\title{
An interoceptive basis for alcohol priming effects
}

\author{
Mateo Leganes-Fonteneau ${ }^{1,2}$ (D) $\cdot$ Marsha E. Bates ${ }^{1,2} \cdot$ Evgeny G. Vaschillo $^{1,2} \cdot$ Jennifer F. Buckman $^{1,2}$
}

Received: 22 October 2020 / Accepted: 8 February 2021 / Published online: 18 February 2021

(C) The Author(s), under exclusive licence to Springer-Verlag GmbH, DE part of Springer Nature 2021

\begin{abstract}
Rationale Alcohol priming can modulate the value of rewards, as observed through the effects of acute alcohol administration on cue reactivity. However, little is known about the psychophysiological mechanisms driving these effects. Here, we examine how alcohol-induced changes in bodily states shape the development of implicit attentional biases and explicit cue reactivity.

Objectives To characterize the interoceptive correlates of alcohol priming effects on alcohol attentional biases and cue reactivity. Methods In a two-session double-blind alcohol administration procedure, participants $(n=31)$ were given a $0.4-\mathrm{g} / \mathrm{kg}$ dose of alcohol or a placebo drink. Cardiovascular responses were measured before and after alcohol administration to observe the effects of alcohol on viscero-afferent reactivity, as indexed through changes in heart rate variability (HRV) at or near $0.1 \mathrm{~Hz}(0.1-\mathrm{Hz}$ HRV). Next, participants completed a modified flanker task to examine implicit alcohol attentional biases and provided subjective valence and arousal ratings of alcohol cues to examine explicit cue reactivity.

Results We found that changes in $0.1-\mathrm{Hz} \mathrm{HRV}$ after alcohol administration positively correlated with attentional biases, and negatively correlated with alcohol valence ratings; blood alcohol content was a null predictor.

Conclusions This is novel evidence that suggests alcohol-induced changes in bodily states may mediate the occurrence of alcohol priming effects and highlights the potentially generative role of interoceptive mechanisms in alcohol-related behaviors. The differential patterns revealed by implicit biases and explicit response tendencies are considered within the context of the dissociation between wanting and liking.
\end{abstract}

Keywords Alcohol priming $\cdot$ Interoception $\cdot$ Heart rate variability $\cdot$ Attentional bias $\cdot$ Alcohol administration

\section{Introduction}

The role of interoceptive signals in addictive behaviors is gaining prominence (Naqvi and Bechara 2010; VerdejoGarcia et al. 2012). Interoceptive signals provide afferent information about the physiological condition of all tissues and organs of the body (Craig 2014) and are key determinants of bodily states (Cameron 2001). The integration, perception, and interpretation of bodily states importantly participate in adaptive cognitive responses (Strigo and Craig 2016) as well

Mateo Leganes-Fonteneau

mateo.leganes@rutgers.edu

Marsha E. Bates

mebates@smithers.rutgers.edu

1 Department of Kinesiology and Health, Rutgers University, New Brunswick, NJ, USA

2 Cardiac Neuroscience Laboratory, Center of Alcohol and Substance Use Studies, Rutgers University, New Brunswick, NJ, USA as in maladaptive addictive behaviors (Gray and Critchley 2007); they also mediate the detection of explicit alcohol effects (Leganes-Fonteneau et al. 2019a).

One of the mechanisms by which bodily states are posited to participate in addictive behaviors is by modulating the value of rewards, a process known as alliesthesia (Paulus et al. 2009). Changes in an individual's internal milieu can change the appeal of a stimulus in a similar way that hunger increases the palatability of food (positive alliesthesia) and satiety decreases it (negative alliesthesia) (Cabanac 1971). Importantly, this internal milieu is subject to intrinsic and extrinsic pressures, such as homeostatic set points and pharmacological challenges, respectively (Avery et al. 2017).

Small doses of a rewarding substance can prime the organism to increase the value of related rewards (Toates 1986). Such priming appears to be of crucial relevance for the understanding of alcohol relapses and clinical prognosis (Rose 2013), as increased salience of alcohol-related stimuli elicited by drinking initiation (when doses are still low) may affect behavior and predict continued drinking (Weinstein and Cox 2006). Although not originally interpreted within the 
framework of interoception and alliesthesia, alcohol priming can be considered in this context. At low levels of intoxication, increases in implicit alcohol attentional biases are observed compared to placebo (Schoenmakers et al. 2008), whereas at higher doses of alcohol, these attentional biases dissipate (Rose and Duka 2006). These findings are consistent with positive and negative alliesthesia, respectively.

The study of interoception and an individual's awareness of their internal milieu requires mechanistically grounded, objective, and sensitive indices of afferent signaling that convey information about body state to the brain. Afferent responses emanating from the cardiovascular system offer a particularly valuable window into the study of bodily states influencing cognitive processes because of an extensive literature detailing the mechanisms that drive heart-brain signaling. The heart and brain maintain continuous and bidirectional information exchange that is measurable as the variability in beat-to-beat intervals in the heart (i.e., heart rate variability (HRV)). There is a robust literature detailing the mechanisms that drive HRV at different oscillations (Task Force 1996). Our laboratory is interested in the variability that occurs specifically at or near $0.1 \mathrm{~Hz}$. This oscillation parallels the inherent $\sim 10$-s cycle of the baroreflex, a body-brain feedback loop that participates in blood pressure regulation and integration of cardiac signals in cognitive-emotional experience (Eddie et al. 2020; Mather and Thayer 2018; Vaschillo et al. 2012). As such, the amplitude of the oscillation observed at or near $0.1 \mathrm{~Hz}$ reflects afferent vagus nerve signaling that relays information about the internal state of the body to interoceptive neural hubs in the brain (Lehrer and Gevirtz 2014). We propose that $0.1-\mathrm{Hz}$ HRV operates in parallel to traditional cardiac interoceptive signals (Critchley et al. 2004), and its measurement can provide novel information about how interoceptive signals shape cognitive function (Leganes-Fonteneau et al. 2021, 2020).

$0.1-\mathrm{Hz}$ HRV also plays a unique role in the study of alcohol-related responses. The magnitude of increase in $0.1-\mathrm{Hz} \mathrm{HRV}$ in the presence of alcohol-related stimuli characterizes alcohol risk factors, such as excessive alcohol use (Mun et al. 2008), and a family history of alcohol use disorder following alcohol administration (Bates et al. 2020). Furthermore, we found that $0.1-\mathrm{Hz}$ HRV reactivity to the presence of alcohol stimuli mapped onto memories for those same stimuli, providing further support for 0.1$\mathrm{Hz} \mathrm{HRV}$ as an interoceptive cardiac signal shaping alcohol cognitive biases (Leganes-Fonteneau et al. 2020). This effect was found only after alcohol administration, suggesting a critical role for positive alliesthesia in the linking between physiological and cognitive alcohol cue reactivity. Moreover, the association was dampened by increased feelings of intoxication, suggesting negative alliesthetic processes may also be at play. Importantly, alcohol administration has a unique and immediate effect on $0.1-\mathrm{Hz}$ HRV (Leganes-Fonteneau et al. 2020). By obtaining
ECG measurements before and after beverage administration (mean blood alcohol concentration, BAC=74 mg/dl), we found decreases in $0.1-\mathrm{Hz} \mathrm{HRV}$ after alcohol administration; sensitive null effects were obtained for participants given a placebo or control beverage. The highfrequency (HF) band of HRV however was affected by all three beverages. Therefore, we propose that $0.1-\mathrm{Hz}$ HRV can be used as a proxy to quantify alcoholinduced changes in bodily states. These changes would in turn allow us to study how alliesthetic processes help shape alcohol cognitive biases.

In this double-blind two-session experiment, ECG measurements were obtained before and $5 \mathrm{~min}$ after alcohol $(0.4 \mathrm{~g} / \mathrm{kg})$ or placebo administration. HRV was measured as the amplitude of the power peak at $0.1 \mathrm{~Hz}$ during a low demand task, in parallel to Leganes-Fonteneau et al. (2020), to obtain an index of bodily changes to alcohol administration. Participants then completed a modified flanker task (with alcohol and neutral stimuli presented as task-irrelevant distractors), which has proven sensitive to priming effects by low alcohol doses $(0.4 \mathrm{~g} / \mathrm{kg}$, Nikolaou et al. 2013a) but has not been assessed in relation to interoceptive cardiac signaling. Subjective picture cue ratings of valence and arousal were included to study explicit responses towards alcohol cues. To our knowledge, subjective ratings have not been previously paired with attentional responses towards alcohol stimuli in the context of an alcohol administration procedure. Thus, this study was designed to characterize how alcohol-induced changes in bodily states modulated the value of alcoholrelated stimuli across implicit (flanker task) and explicit (cue ratings) cognitive domains (Wiers and Stacy 2006).

To demonstrate successful task effects, we sought to replicate the observation that longer reaction time latencies would be observed when alcohol stimuli, as compared to neutral stimuli, appeared as distractors in the flanker task. We further sought to replicate the observation that this effect would be more pronounced during high cognitive load trials (Leganes-Fonteneau et al. 2019b; Nikolaou et al. 2013b). Because of the deleterious effect of alcohol on flanker task performance during high cognitive load (Marinkovic et al. 2013), the detection of attentional biases could be restricted to low cognitive load trials after alcohol administration (Nikolaou et al. 2013a). The main hypotheses, however, target the larger, novel design of this experiment. Specifically, we expected that the modulation of bodily states by alcohol administration, indexed as changes in $0.1-\mathrm{Hz} \mathrm{HRV}$, would predict alcohol attentional biases and explicit picture cue ratings. Due to a lack of prior studies on this topic, we did not hypothesize the directionality of these relationships, but the relationship between $0.1-\mathrm{Hz} \mathrm{HRV}$ and attentional biases could depend on cognitive load and beverage. 


\section{Methods}

\section{Participants}

Participants $(n=31$, mean age $=23.52, \mathrm{SD}=4.05,16$ males $)$ were recruited from fliers posted on or near Rutgers University, NJ, USA. Participants were eligible if they drank more than three alcohol units per week in the past 3 months, were at least 21 years old, and did not report a history of learning disability, psychiatric disorders, treatment for substance use disorder, current AUD, or regular (weekly) illicit or prescription drug use. Additionally, pregnant women and participants with a body mass index (BMI) 20\% over- or under-weight were excluded. Upon arrival to the laboratory, weight was confirmed as well as pregnancy status using a pregnancy test. Participants were compensated for their participation in the study (USD 35-45).

Three participants completed only 1 session (alcohol session only, $n=2$; placebo session only, $n=1$ ) due to premature study termination related to COVID-19 and their data were not included in analyses. Furthermore, ECG data for three participants were not available due to technical errors (one in the alcohol condition, two in the placebo condition), but their available data but was included. An a priori power analysis was performed for sample size estimation, based on correlational data using the modified flanker task (Nikolaou et al. $2013 \mathrm{~b}$ ) that yielded an effect size $=0.451$. With an alpha $=0.05, n=26$ participants would generate a power $=0.80$, and $n=35$ a power $=0.90$. The recruited sample size is thus adequately powered (0.85) for the main objective of our study.

\section{Questionnaires}

Questionnaires evaluating alcohol use are presented in the Appendix in the Supplementary information.

\section{Beverage administration}

In a double-blind alcohol administration procedure, participants completed two sessions, one in which they were given an alcohol beverage and one in which they were given the placebo beverage; the order of the conditions was counterbalanced accounting for gender. The alcohol beverage consisted of $0.4 \mathrm{~g} / \mathrm{kg}$ of alcohol (equivalent to $\sim 2$ standard alcohol drinks for a $70-\mathrm{kg}$ person), obtained from 190-proof Everclear mixed with sugar-free Schweppes tonic water and 5 drops of angostura bitters to make up a 200-ml solution. The placebo beverage consisted of $200 \mathrm{ml}$ of Schweppes with 5 drops of Angostura bitters. The solution was served in 5 plastic glasses $(40 \mathrm{ml}$ each) and alcohol was sprinkled over the serving tray for olfactory cues. Participants had 2 min to drink each serving (drink times paced via MATLAB). BAC was measured 2 times using an Alco-Pro Alco-Sensor FST, starting 10 min after the beverage consumption was completed.

\section{Physiological reactivity}

Participants' cardiac physiology was measured before beverage administration $\left(t_{0}\right)$ and 5 min after $\left(t_{1}\right)$ using the vanilla task (Jennings et al. 2007). Participants were instructed to sit while a series of colored squares were presented on the screen. Each square appeared for $10 \mathrm{~s}(0.1-\mathrm{Hz}$ presentation). This was repeated 30 times for a total period of $5 \mathrm{~min}$. Participants were instructed to silently count how many "blue" $\left(t_{0}\right)$ or "red" $\left(t_{1}\right)$ squares they observed, although responses were irrelevant.

Electrocardiogram (ECG) measures were obtained using Thought Technology Infiniti hardware and software (Thought Technology Inc. SA9306M). Data were obtained at a $2000-\mathrm{Hz}$ sampling rate and exported using WinCPRS software (Absolute Alien Oy, Turku, Finland) to measure Rwave to R-wave intervals (RRI) of ECG. Artifacts and missed or irregular beats were manually modified prior to the analysis. Beat-to-beat data were transformed into equidistant waveform processes through cubic interpolation and 4-Hz resampling. Fourier spectra of RRI were then calculated for $0.1 \mathrm{~Hz}$ and HF HRV. The $0.1-\mathrm{Hz}$ HRV index was the main physiological measure of interest in this report and was calculated as the maximum amplitude of the RRI spectral power (Buckman et al. 2010; Mun et al. 2008; Vaschillo et al. 2008) within a narrow range surrounding the $0.1-\mathrm{Hz}$ frequency $(0.075$ to $0.108 \mathrm{~Hz}$ ). This index is thought to reflect individual differences in the amplitude of baroreflex responsivity to stimulation (Vaschillo et al. 2002, 2006) and thus serves as a proxy for the evaluation of viscero-afferent reactivity (LeganesFonteneau et al. 2020). The HF HRV index was calculated as total power in the high-frequency band of the RRI spectrum $(0.15-0.5 \mathrm{~Hz})$. HF HRV is commonly equated with efferent vagal functioning and was included as a "control" cardiovascular measure that was not expected to correspond with interoceptive signaling.

\section{Modified flanker task}

This task examines the interference of alcohol cues, compared to neutral and control pictures, on attention and cognitive control (Nikolaou et al. 2013b). On each trial, participants had to resolve a congruent flanker or an incongruent flanker. Behind the flanker arrows was a task-irrelevant cue: either an alcohol, neutral, or control (plain gray) stimulus that was randomly presented during the task in a 240-trial block. (See Fig. 1, Appendix in the Supplementary information, and previous research (Nikolaou et al. 2013b for a detailed task description.)

\section{Cue reactivity task}

During both sessions, participants were presented with two picture cue blocks of 30 pictures each: alcohol or neutral. 
Stimulus $(500 \mathrm{~ms})$

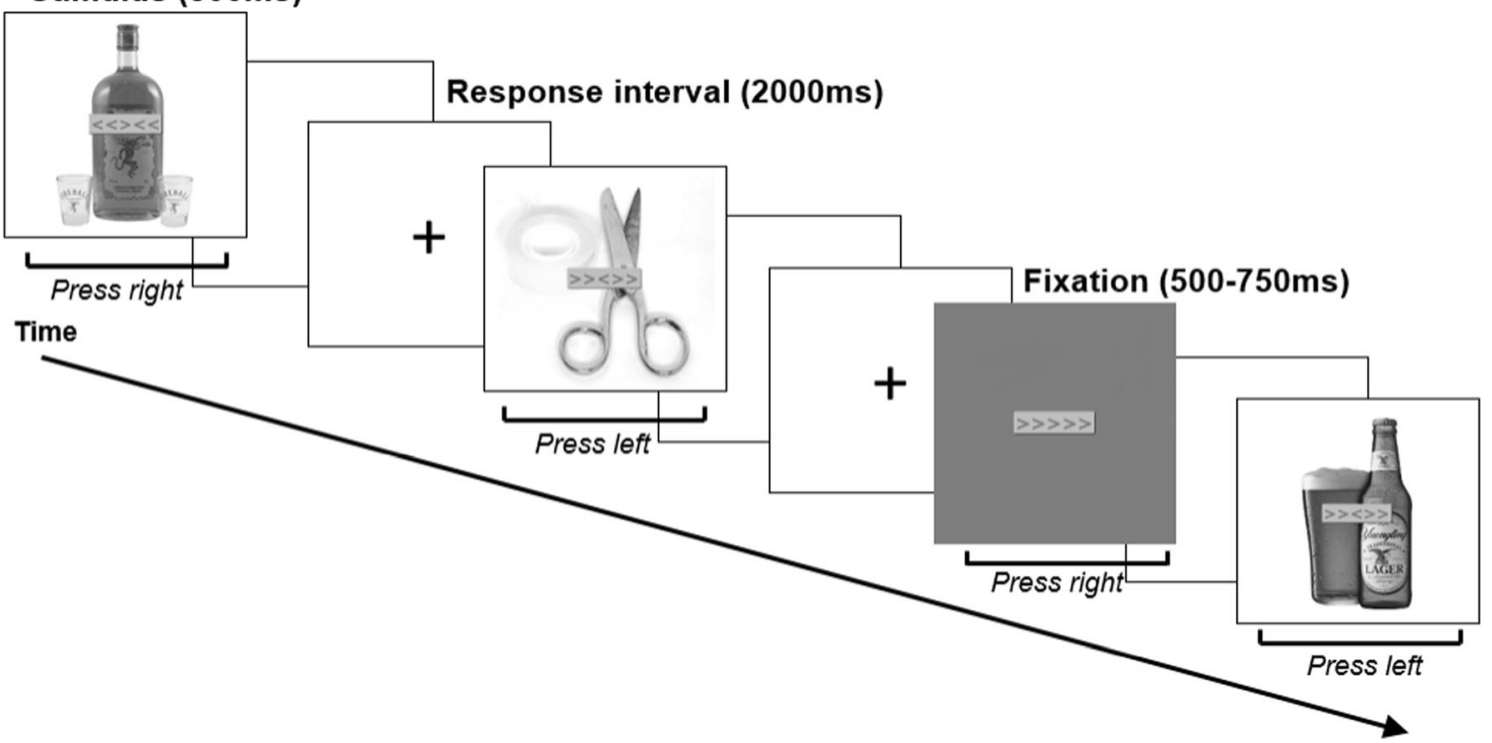

Fig. 1 Modified flanker task-participants responded depending on the direction of the central arrow, which could be surrounded by congruent or incongruent flankers. Alcohol, neutral, or control (gray background) stimuli were randomly presented as task-irrelevant distractors

For each cue type, 60 stimuli were selected and randomly divided into two 30 picture blocks so that pictures presented during session 1 and session 2 were unique for each participant.

The alcohol cues consisted of people consuming different beverages, drinks being served, and pictures of naturalistic drinking environments. Neutral cues consisted of images of plants and people gardening or reading and were matched as much as possible to the features of alcohol stimuli (close up objects, number of people together in the scene (Bates et al. 2019)). Each picture remained on the screen for $5 \mathrm{~s}$ followed by a 5 -s inter-stimulus interval during which participants rated the valence (positive/negative) and arousal (not at all/very) for each picture cue on a visual analog scale.

All stimuli for the cue reactivity and flanker task are available on Open Science Framework to ensure experimental transparency (https://osf.io/gzp3y/?view_only= cf0742461f4844a2a5553068dfe249a7).

\section{Procedure}

This study was approved by the Rutgers University Arts and Sciences Institutional Review Board for the Protection of Human Subjects Involved in Research and all participants provided written informed consent. All tasks were programmed using MATLAB Psychtoolbox.

Eligible participants completed both experimental sessions at least 1 week apart and were instructed to have a low-fat breakfast and to not eat for the 2-h period prior to the experiment. They were also asked to not drink alcohol for $24 \mathrm{~h}$ before the sessions. In the first session, after the consenting process, participants completed the questionnaires assessing traits and alcohol consumption and took part in an interoceptive awareness measurement (not presented here). At $t_{0}$, participants were connected to the ECG equipment and completed the first physiological baseline task ("blue" vanilla task). Participants were administered a beverage according to the procedure described above. After a 5-min rest, a second ECG measurement $\left(t_{1}\right)$ was taken ("red" vanilla task) followed by a BAC measurement (mean BAC $t_{1}=58 \mathrm{mg} / \mathrm{dl}, \mathrm{SD}=18$ ). Finally, participants completed the modified flanker task and the cue reactivity task. ECG measures were obtained during the cue reactivity task (data not presented). BAC was collected again at $t_{2}$ (mean BAC $t_{2}=57 \mathrm{mg} / \mathrm{dl}, \mathrm{SD}=13$ ). The protocol was designed ensure all tasks were completed on the ascending limb, near peak BAC. Participants remained in the facilities until they reached a BAC $<3 \mathrm{mg} / \mathrm{dl}$. (See Fig. 2 for a description of the protocol.)

\section{Descriptive analyses}

Trait questionnaire scores (Table A1 in the Supplementary information), flanker scores, explicit cue ratings, and the effect of alcohol on HRV are presented to characterize the sample in the Appendix section in the Supplementary information. Flanker scores and explicit cue ratings are analyzed using planned comparisons. Paired-samples $t$ tests assessed the effect of alcohol administration on HRV.

\section{Hypothesis testing}

Flanker accuracy (number accurately identified) and latencies (reaction time for accurate trials) were computed for each distractor stimulus type (alcohol, neutral, control) and 
Fig. 2 Description of the laboratory experimental protocol

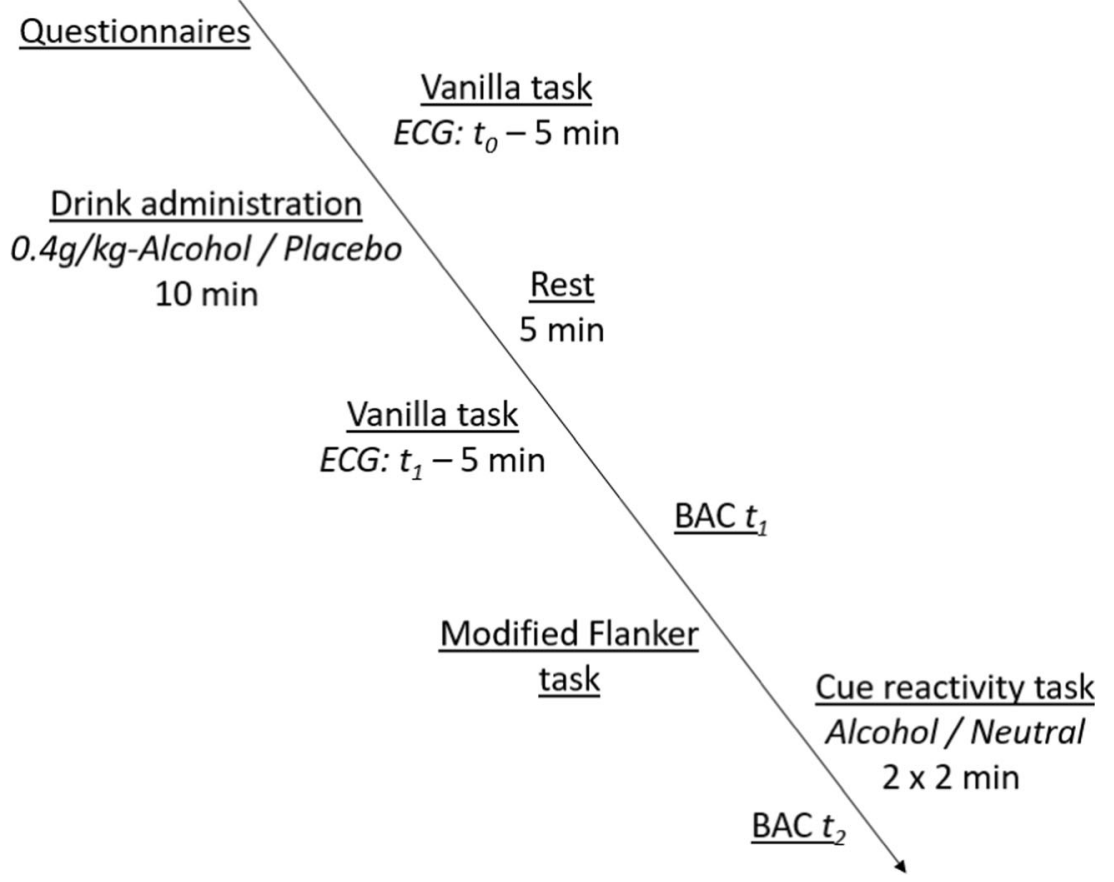

cognitive load (congruent, incongruent) combination. Accuracy scores on incongruent control trials were examined to exclude any participant with scores $<0.70$ (no exclusions).

The main purpose of this experiment was to determine if alcohol-induced changes in $0.1-\mathrm{Hz}$ HRV predict attentional biases and explicit ratings for alcohol stimuli. Physiological change (Physio $\Delta$ ) was computed as the change in $0.1 \mathrm{~Hz}$ and HF HRV from before to after beverage administration $\left(t_{1}-t_{0}\right)$. HRV values were log-transformed. Higher scores are indicative of less reduction of HRV following beverage administration. Flanker scores were computed as the difference in latencies (alcohol-neutral) for congruent and incongruent trials separately. This allowed us to index attentional biases towards alcohol (congruent trials), and the interference in cognitive control (incongruent trials). Higher scores are indicative of more attentional bias and greater interference by alcohol stimuli.

A series of linear mixed models with session (alcohol vs. placebo) and 0.1-Hz HRV Physio $\Delta$ as fixed factors and BAC at $t_{1}$ as a covariate were conducted on congruent and incongruent flanker effects separately. Significant interactions were followed by simple slope analyses examining the effect of 0.1 $\mathrm{Hz}$ HRV Physios on flanker effects for each session. For valence and arousal ratings, difference scores were computed (alcohol-neutral ratings) and introduced as DVs in analogous linear mixed models. To further demonstrate the specificity of the observed results relative to viscero-afferent/interoceptive signaling, we repeated the analyses using changes in HF HRV instead of changes in $0.1-\mathrm{Hz} \mathrm{HRV}$; we did not predict any significant relationships. False discovery rates based on the Benjamini-Hochberg method (https://tools.carbocation.com/
FDR) were used to control for multiple comparisons in the linear mixed models, separately for those including $0.1-\mathrm{Hz}$ HRV and HF HRV as Physios; all $p$ values for the interactions are adjusted unless otherwise indicated.

\section{Results}

Analyses of flanker scores (Table A2 in the Supplementary information) and explicit cue ratings (Table A3 in the Supplementary information) demonstrate task success and replicate prior observations. We did not replicate a significant effect of alcohol on 0.1-Hz HRV (Figure A1 in the Supplementary information), but there was a substantial heterogeneity in individual responses to alcohol.

\section{Alcohol-induced cardiovascular states and attentional biases}

For congruent flanker scores, there was a significant interaction between $0.1-\mathrm{Hz}$ HRV Physios and session $(F(1,53)=6.052, p=.034)$, whereas BAC had no significant effect $(F(1,53)=0.009, p=.926)$. Simple slope analyses show that in the alcohol condition $0.1-\mathrm{Hz} \mathrm{HRV}$ positively correlated with congruent flanker scores $(\beta=.604, \mathrm{SE}=0.223$, $t(53)=2.713, p=.009)$, whereas no significant effects were observed for placebo $(t(53)=-0.813, p=.417)$. Figure 3 shows the variability in the distribution of participants' congruent flanker scores and how those with less reduction in $0.1-\mathrm{Hz}$ HRV after alcohol administration displayed more attentional biases towards alcohol stimuli. 


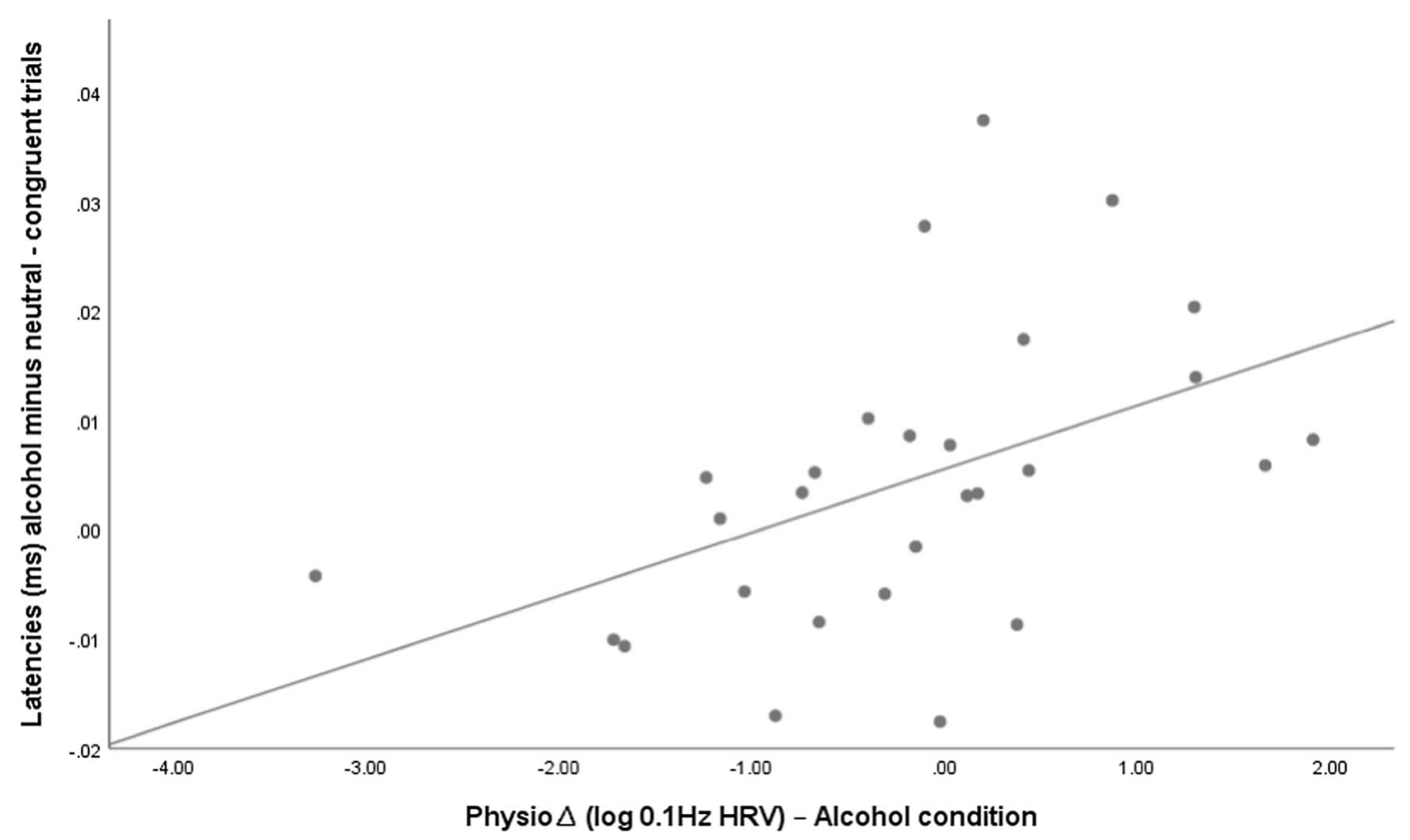

Fig. 3 Alcohol condition and the relationship between flanker congruent score and 0.1-Hz HRV: Less reduction in 0.1-Hz HRV after alcohol administration positively predicted alcohol attentional biases

A parallel linear mixed model using incongruent flanker scores as a DV was non-significant $(F(1,53)=0.197, p=.877)$. Using HF HRV Physio $\Delta$ as a covariate, no significant interactions were observed for congruent $(F(1,53)=0.481, p=.655)$ or for incongruent $(F(1,53)=1.567, p=.864)$ flanker scores.

\section{Alcohol-induced cardiovascular states and explicit ratings}

Valence difference scores were inverse-transformed due to violations of normality of the residuals. The linear mixed model including $\mathrm{BAC}$ as a covariate revealed no significant interaction between 0.1-Hz HRV Physio $\Delta$ and session ( $p=.096$ ), nor a significant effect of BAC $(p=.818)$. When the model was tested excluding $\mathrm{BAC}$ scores, there was a significant interaction between 0.1-Hz HRV Physio $\Delta$ and session $(F(1,31)=7.476$, $p=.0404)$. Simple slope analyses show that in the alcohol condition, $0.1-\mathrm{Hz} \mathrm{HRV}$ negatively correlated with valence scores $(\beta=.196, \mathrm{SE}=0.077, t(37)=2.550, p=.015)$, whereas no significant effects were observed for placebo $(t(37)=-1.228, p=.227)$.

For arousal difference scores, there was no significant interaction between $0.1-\mathrm{Hz}$ HRV Physio $\Delta$ and session $(F(1,48)=0.0396, p=.843)$. There was a significant main effect of $0.1-\mathrm{Hz}$ HRV Physio $\Delta$ on arousal difference scores across the board $(F(1,48)=7.484 p=.009)$, signified by a negative correlation between $0.1-\mathrm{Hz}$ HRV Phsio $\Delta$ and arousal difference scores $(r(51)=-0.336, p=.014)$. Figure $4 \mathrm{~b}$ and $\mathrm{c}$ show the effects separately for alcohol and placebo despite the lack of significant differences between sessions.
Using HF HRV Physio $\Delta$ as a covariate, no significant interactions were observed, neither for valence $(F(1,48)=0.725$, $p=.796)$, nor for arousal difference scores $(F(1,48)=-0.159$, $p=.874)$.

We used R to assess assumptions of linearity, homogeneity of variance and normality, and qqmath with a threshold of 0.05 to remove multivariate outliers. Removal of outliers did not modify the significance of the interactions except in the case of valence scores, for which the removal of one outlier decreased the significance of the main model from $p=.010$ to $p=.17$ (unadjusted). The simple slope analysis showed that the interaction between $0.1-\mathrm{Hz} \mathrm{HRV}$ and valence scores remained significant for the alcohol condition $(p=.019)$ and nonsignificant for the placebo condition $(p=.57)$.

\section{Discussion}

This study examined how alliesthetic processes influence the value of alcohol-related stimuli. Our results are in line with

Fig. 4 Relationship between 0.1-Hz HRV Physios and subjective cue reactivity. a Less reduction in $0.1-\mathrm{Hz} \mathrm{HRV}$ after alcohol administration was negatively associated with valence ratings of alcohol stimuli relative to neutral stimuli. b Less reduction in $0.1-\mathrm{Hz} \mathrm{HRV}$ after alcohol administration was negatively associated with arousal ratings of alcohol stimuli relative to neutral stimuli. c Less reduction in $0.1-\mathrm{Hz} \mathrm{HRV}$ after placebo administration was negatively associated with arousal ratings of alcohol stimuli relative to neutral stimuli 

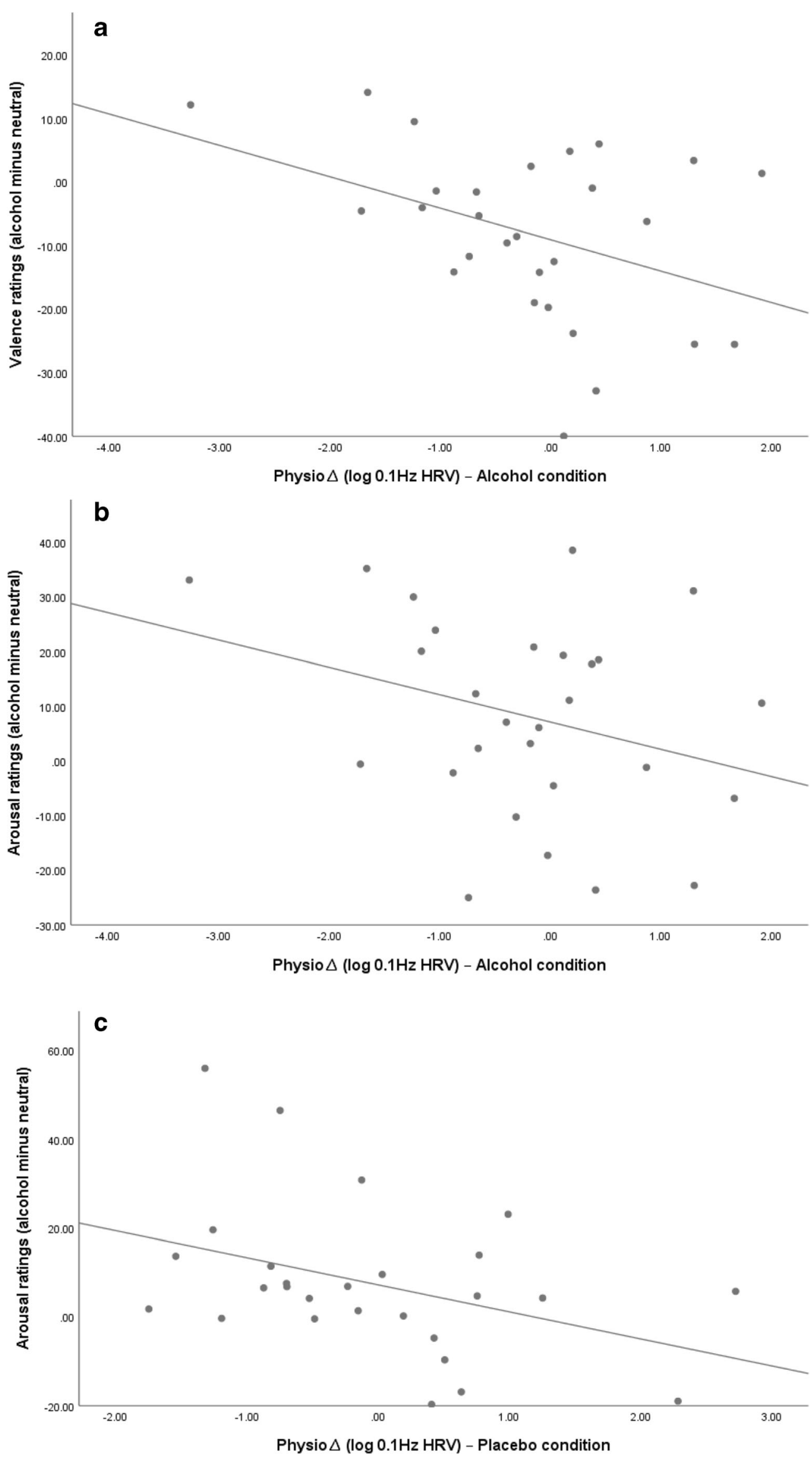
previous findings that alcohol priming modulates cognitive biases on the ascending limb of the BAC curve. They also extend that literature by offering a new mechanistic perspective on the role of bodily states in this effect. We found that changes in 0.1-Hz HRV after consumption of an alcohol beverage positively predicted implicit alcohol attentional biases as measured with a modified flanker task; conversely, it negatively predicted explicit valence ratings of alcohol cues. It is noteworthy that BAC at the time of task completion did not explain these results. This implies that alcohol-induced changes on the cardiovascular system, and not blood alcohol concentration per se, were responsible for the observed modulation of alcohol-related cognitive biases. This pattern of results reveals the potential significance of alliesthetic mechanisms in alcohol priming.

We consider $0.1-\mathrm{Hz} \mathrm{HRV}$ as a measure of viscero-afferent reactivity, indexing interoceptive cardiovascular signals (Leganes-Fonteneau et al. 2020); changes in these interoceptive signals represent the modulation of the internal milieu of the subject by alcohol administration to modify reward values (Toates 1986). Higher doses of alcohol have been shown to decrease all indices of HRV (mean BAC $\sim 75 \mathrm{mg} / \mathrm{dl}$, LeganesFonteneau et al. 2020; Vaschillo et al. 2008), but we did not replicate this finding with the current lower dose. Here, we observed large variability in participants' $0.1-\mathrm{Hz}$ HRV reactivity to the current dose of alcohol that gave rise to a lower BAC; some participants showed the expected decreases following alcohol administration whereas others did not. Participants with less $0.1-\mathrm{Hz}$ dampening after alcohol administration obtained higher alcohol flanker latency scores in congruent trials, indicative of greater attentional bias to alcohol stimuli. BAC was a sensitively null predictor in these models. Change in HF HRV did not significantly correlate with any index of cue reactivity. This strengthens growing evidence for the uniqueness of 0.1$\mathrm{Hz} \mathrm{HRV} \mathrm{as} \mathrm{a} \mathrm{measure} \mathrm{of} \mathrm{alcohol-induced} \mathrm{changes} \mathrm{in} \mathrm{bodily}$ states in relation to cognitive processing. This also supports previous evidence for the predictive and discriminative validity of 0.1-Hz HRV compared to other HRV indices in relation to emotional reactivity (Vaschillo et al. 2008).

In addition to $0.1-\mathrm{Hz} \mathrm{HRV}$ reactivity predicting implicit attentional biases, this measure of viscero-afferent reactivity was negatively associated with explicit valence ratings of alcohol cues. Although we hypothesized that changes across all measures would be in the same direction, the distinction between the implicit and explicit components of alcohol cue reactivity has been demonstrated in the past (Wiers et al. 2016) and has the mismatch between implicit responses, measured using a flanker task, and explicit ratings of pleasantness towards reward conditioned stimuli (Leganes-Fonteneau et al. 2019b). The present findings (Physio $\Delta$ positively predicting attentional biases and negatively valence ratings) suggest that the physiological effects of alcohol administration exacerbate this differentiation, further disconnecting implicit processes that represent involuntary responses towards alcohol stimuli, from explicit evaluations that are constrained by conscious representations (de Wit et al. 2018; Wiers et al. 2021).

We note that the observation of lower valence ratings but higher arousal ratings for alcohol stimuli compared to neutral stimuli in both alcohol and placebo conditions was not expected. To our knowledge, few studies have directly examined differences in explicit hedonic responses towards alcohol cues under alcohol/placebo administration, despite subjective ratings being a widely used measure (Pool et al. 2016; Pulido et al. 2010). Higher valence ratings for alcohol stimuli compared to neutral regardless of beverage condition using a similar stimulus set have been found (Nguyen-Louie et al. 2016). However, in that case, stimuli were presented during the ascending limb right after the administration of a higher alcohol dose. The role of dose and time in explicit valence ratings warrants further exploration.

It is interesting to consider our findings within an incentive salience framework, wherein "wanting" and "liking" are two distinct components of reward processing (Berridge and Robinson 2003). In that context, addictive disorders are characterized by increases in "wanting," understood as motivational responses (i.e., reward-related attentional biases, Anderson and Yantis 2013), and decreases in "liking," the hedonic characteristics of rewards. Although there is abundant evidence for this effect in animal research, empirical behavioral evidence in humans is limited (Arulkadacham et al. 2017). Earlier reports pointed towards the possibility that implicit and explicit components of cue reactivity would allow a clearer distinction between "wanting" and "liking" (Wiers et al. 2002). We speculate that the present implicit and explicit (i.e., subjective) reactions to alcohol could map onto the constructs of "wanting" and "liking" respectively. This distinction between "wanting" and "liking" is observable such that alcohol stimuli consistently generated preferential attentional responses and decreased valence ratings relative to neutral stimuli in both the placebo and alcohol conditions (see Appendix in the Supplementary information). Physiological states have been linked to the dissociation of "wanting" and "liking," albeit in the context of food administration (Stevenson et al. 2017). Our methodology allows quantifying the extent to which changes in bodily states might magnify this dissociation and help clarify it. Specifically, less dampening of 0.1-Hz HRV during intoxication was associated with greater implicit attentional bias, as measured with the modified flanker task, which we liken to an increased "wanting." Less $0.1-\mathrm{Hz}$ HRV dampening by intoxication also was associated with less explicit positive ratings of alcohol stimuli in a cue reactivity task, which we liken to a decreased "liking."

Future studies that directly test this hypothesis in different populations of drinkers, particularly those with AUD, are needed before strong conclusions can be made, as the dissociation between "wanting" and "liking" is supposed to emerge as trajectories of use accelerate. Our findings could also be 
methodologically biased because "wanting" is observable implicitly and "liking" explicitly. A direct replication of the dissociation using only explicit or implicit techniques would be challenging, but highly informative. We also speculate that, in humans, hedonic responses ("liking") may only be measurable explicitly (i.e., it may not possible to like something without knowing it), whereas behavioral tendencies ("wanting") may only (or primarily) have an implicit component (LeganesFonteneau et al. 2019b). That debate exceeds the focus of this report but is a compelling direction for future research.

Finally, it is noteworthy that the present flanker results in the placebo condition (see Appendix in the Supplementary information) directly replicated previous findings (Nikolaou et al. 2013b): alcohol stimuli increased interference in cognitive control during incongruent trials (when no alcohol is administered). In the alcohol condition, we also replicated previous findings of attentional biases observed in congruent trials with a $0.4 \mathrm{~g} / \mathrm{kg}$ dose (Nikolaou et al. 2013a), whereas insensitive results were obtained in incongruent trials, signifying the deleterious effect of alcohol administration on performance and cognitive processing (Marinkovic et al. 2013). This could be interpreted as an alcohol myopia effect (Steele and Josephs 1990), by which alcohol intoxication reduces participants' cognitive resources (Watson et al. 2020), forcing them to focus on the main task (resolving the flanker) and to ignore task-irrelevant distractors (alcohol stimuli). Congruent flanker trials would be easier to resolve, and hence, alcohol distractors would have a better chance to generate attentional interference. This hypothesis also warrants further study.

To summarize, the results show that alcohol-induced changes in bodily states can modulate reward processing. This is one of the first successful examinations of the physiological mechanisms correlating alcohol priming effects in humans and at the same time an empirical demonstration of the hypothesized alliesthetic processes that shape drug-related behaviors (Paulus et al. 2009). The importance of interoceptive signals and bodily states in reward processing had long been hypothesized (Gray and Critchley 2007; Verdejo-Garcia et al. 2012), and the present results align with recent evidence from our research group showing that memories for alcohol stimuli in high-risk drinkers depend on bodily reactivity towards those same stimuli after alcohol administration (Leganes-Fonteneau et al. 2020). Understanding how physiological and interoceptive mechanisms shape reward processing is not only relevant for the basic understanding of addictive disorders but can also help to shape novel treatment tools, focused on the link between interoception and cognition.

Supplementary Information The online version contains supplementary material available at https://doi.org/10.1007/s00213-021-05796-w.

Acknowledgments We wish to thank Dr. Kyriaki Nikolaou for her suggestions on data analysis and Shariar Islam for his assistance on data collection.
Funding This research was supported in part by grants R01AA023667 and K02AA025123 from the National Institute of Alcohol Abuse and Alcoholism.

Data availability Data will be made available upon paper acceptance on OSF.

\section{Declarations}

Conflict of interest The authors declare no competing interests.

\section{References}

Anderson BA and Yantis S (2013) Persistence of value-driven attentional capture. J Exp Psychol Hum Percept Perform 39(1). NIH Public Access: 6-9. https://doi.org/10.1037/a0030860.

Arulkadacham LJ, Richardson B, Staiger PK, et al. (2017) Dissociation between wanting and liking for alcohol and caffeine: a test of the Incentive Sensitisation Theory. J Psychopharmacol (Oxford, England) 31(7). SAGE Publications Ltd: 927-933. https://doi.org/ 10.1177/0269881117711711.

Avery JA, Burrows K, Kerr KL, et al. (2017) How the brain wants what the body needs: the neural basis of positive alliesthesia. Neuropsychopharmacology 42(4). Nature Publishing Group: 822830. https://doi.org/10.1038/npp.2016.128.

Bates ME, Lesnewich LM, Uhouse SG, et al. (2019) Resonance-paced breathing alters neural response to visual cues: proof-of-concept for a neuroscience-informed adjunct to addiction treatments. Front Psychiatry 10. Frontiers Media S.A.: 624 https://doi.org/10.3389/ fpsyt.2019.00624.

Bates ME, Mun EY, Buckman JF, et al. (2020) Getting to the heart of low sensitivity to alcohol: context moderates low cardiovascular response to alcohol in persons with a family history of alcohol use disorder. Alcohol Clin Exp Res 44(3). Blackwell Publishing Ltd: 589-599. https://doi.org/10.1111/acer.14293.

Berridge KC, Robinson TE (2003) Parsing reward. Trends Neurosci 26(9):507-513. https://doi.org/10.1016/S0166-2236(03)00233-9

Buckman JF, White HR and Bates ME (2010) Psychophysiological reactivity to emotional picture cues two years after college students were mandated for alcohol interventions. Addict Behav 35(8). Pergamon: 786-790. https://doi.org/10.1016/J.ADDBEH.2010.03. 017.

Cabanac M (1971) Physiological role of pleasure. Science (New York, N.Y.) 173(4002): 1103-7. Available at: http://www.ncbi.nlm.nih. gov/pubmed/5098954 (accessed 19 June 2018).

Cameron OG (2001) Interoception: the inside story - a model for psychosomatic processes. Psychosomatic Medicine 63(5). Psychosomatic Medicine: 697-710. Available at: https://insights. ovid.com/psychosomatic-medicine/psme/2001/09/000/ interoception-inside-story-model-psychosomatic/1/00006842 (accessed 1 November 2017).

Craig A (2014) How do you feel?: an interoceptive moment with your neurobiological self.

Critchley HD, Wiens S, Rotshtein P, et al. (2004) Neural systems supporting interoceptive awareness. Nat Neurosci 7(2). Nature Publishing Group: 189-95. https://doi.org/10.1038/nn1176.

de Wit H, Epstein DH, Preston KL (2018) Does human language limit translatability of clinical and preclinical addiction research? Neuropsychopharmacology Nat Publ Group 43:1985-1988. https://doi.org/10.1038/s41386-018-0095-8

Eddie D, Bates ME and Buckman JF (2020) Closing the brain-heart loop: towards more holistic models of addiction and addiction recovery. 
Addict Biol. Blackwell Publishing Ltd. : https://doi.org/10.1111/ adb. 12958 .

Gray MA and Critchley HD (2007) Interoceptive basis to craving. Neuron 54(2). Cell Press: 183-186. Available at: https://www. sciencedirect.com/science/article/pii/S0896627307002474 (accessed 8 September 2017)

Jennings JR, Kamarck T, Stewart C, Eddy M, Johnson P (2007) Alternate cardiovascular baseline assessment techniques: vanilla or resting baseline. Psychophysiology 29(6):742-750. https://doi.org/10. 1111/j.1469-8986.1992.tb02052.x

Leganes-Fonteneau M, Cheang Y, Lam Y, et al. (2019a) Interoceptive awareness is associated with acute alcohol-induced changes in subjective effects. Pharmacol Biochem Behav 181. Elsevier: 69-76. https://doi.org/10.1016/J.PBB.2019.03.007.

Leganes-Fonteneau Mateo, Nikolaou K, Scott R, et al. (2019b) Knowledge about the predictive value of reward conditioned stimuli modulates their interference with cognitive processes. Learning \& Memory (Cold Spring Harbor, N.Y.) 26(3). Cold Spring Harbor Laboratory Press: 66-76. https://doi.org/10.1101/lm.048272.118.

Leganes-Fonteneau M, Buckman J, Pawlak A, et al. (2020) Interoceptive signaling in alcohol cognitive biases: role of family history and alliesthetic components. Addict Biol. John Wiley \& Sons, Ltd. DOI: https://doi.org/10.1111/adb.12952

Leganes-Fonteneau M, Buckman J, Islam S et al (2021) The cardiovascular mechanisms of interoceptive awareness: effects of resonance breathing. Int J Psychophysiol Stage 1 Re (in press)

Lehrer PM, Gevirtz R (2014) Heart rate variability biofeedback: how and why does it work? Frontiers in Psychology 5. Frontiers 756. https:// doi.org/10.3389/fpsyg.2014.00756

Marinkovic K, Rickenbacher E, Azma S, Artsy E, Lee AKC (2013) Effects of acute alcohol intoxication on saccadic conflict and error processing. Psychopharmacology 230(3):487-497. https://doi.org/ 10.1007/s00213-013-3173-y

Mather M, Thayer J (2018) How heart rate variability affects emotion regulation brain networks. Current opinion in behavioral sciences 19. NIH Public Access: 98-104. https://doi.org/10.1016/j.cobeha. 2017.12.017

Mun EY, von Eye A, Bates ME, et al. (2008) Finding groups using model-based cluster analysis: heterogeneous emotional selfregulatory processes and heavy alcohol use risk. Dev Psychol 44(2). NIH Public Access: 481-95. https://doi.org/10.1037/00121649.44.2.481

Naqvi NH, Bechara A (2010) The insula and drug addiction: an interoceptive view of pleasure, urges, and decision-making. Brain Struct Funct 214(5-6):435-450. https://doi.org/10.1007/s00429-0100268-7

Nguyen-Louie TT, Buckman JF, Ray S, et al. (2016) Drinkers' memory bias for alcohol picture cues in explicit and implicit memory tasks. Drug Alcohol Depend 160. Elsevier: 90-96. https://oi.org/10. 1016/J.DRUGALCDEP.2015.12.033.

Nikolaou K, Field M, Critchley H, et al. (2013a) Acute alcohol effects on attentional bias are mediated by subcortical areas associated with arousal and salience attribution. Neuropsychopharmacology 38(7). Nature Publishing Group: 1365-1373. https://doi.org/10.1038/npp. 2013.34.

Nikolaou K, Field M, Duka T (2013b) Alcohol-related cues reduce cognitive control in social drinkers. Behav Pharmacol 24(1):29-36. https://doi.org/10.1097/FBP.0b013e32835cf458

Paulus MP, Tapert SF and Schulteis G (2009) The role of interoception and alliesthesia in addiction. Pharmacol Biochem Behav 94(1). Elsevier: 1-7. https://doi.org/10.1016/J.PBB.2009.08.005.

Pool E, Sennwald V, Delplanque S, Brosch T, Sander D (2016) Measuring wanting and liking from animals to humans: a systematic review. Neurosci Biobehav Rev 63:124-142. https://doi.org/10. 1016/j.neubiorev.2016.01.006
Pulido C, Brown SA, Cummins K, et al. (2010) Alcohol cue reactivity task development. Addict Behav 35(2). Pergamon: 84-90. https:// doi.org/10.1016/j.addbeh.2009.09.006.

Rose AK (2013) Substance priming. In: The Wiley-Blackwell Handbook of Addiction Psychopharmacology. Wiley-Blackwell, pp. 435-458. https://doi.org/10.1002/9781118384404.ch16

Rose AK, Duka T (2006) Effects of dose and time on the ability of alcohol to prime social drinkers. Behav Pharmacol 17(1):61-70. https://doi.org/10.1097/01.fbp.0000189814.61802.92

Schoenmakers T, Wiers RW and Field M (2008) Effects of a low dose of alcohol on cognitive biases and craving in heavy drinkers. Psychopharmacology 197(1). Springer-Verlag: 169-178. https:// doi.org/10.1007/s00213-007-1023-5.

Steele CM, Josephs RA (1990) Alcohol myopia: its prized and dangerous effects. Am Psychol 45(8):921-933. https://doi.org/10.1037/0003066X.45.8.921

Stevenson RJ, Francis HM, Attuquayefio T, et al. (2017) Explicit wanting and liking for palatable snacks are differentially affected by change in physiological state, and differentially related to salivation and hunger. Physiol Behav 182. Elsevier Inc.: 101-106. https://doi.org/ 10.1016/j.physbeh.2017.10.007.

Strigo IA and Craig AD (Bud) (2016) Interoception, homeostatic emotions and sympathovagal balance. Philosophical Transactions of the Royal Society B: Biological Sciences 371(1708). The Royal Society: 20160010. https://doi.org/10.1098/rstb.2016.0010.

Task Force O and Of the ES of C and the NAS (1996) Heart rate variability. Standards of measurement, physiologic interpretation, and clinical use. Circulation 93(1043-1065).

Toates FM (Frederick M. (1986) Motivational systems. Cambridge University Press.

Vaschillo E, Lehrer P, Rishe N, et al. (2002) Heart rate variability biofeedback as a method for assessing baroreflex function: a preliminary study of resonance in the cardiovascular system. Appl Psychophysiol Biofeedback 27(1): 1-27. Available at: http:/www. ncbi.nlm.nih.gov/pubmed/12001882 (accessed 21 March 2019).

Vaschillo EG, Vaschillo B and Lehrer PM (2006) Characteristics of resonance in heart rate variability stimulated by biofeedback. Appl Psychophysiol Biofeedback 31(2). Springer US: 129-142. https:// doi.org/10.1007/s10484-006-9009-3.

Vaschillo EG, Bates ME, Vaschillo B, et al. (2008) Heart rate variability response to alcohol, placebo, and emotional picture cue challenges: effects of $0.1-\mathrm{Hz}$ stimulation. Psychophysiology 45(5). Wiley/ Blackwell (10.1111): 847-858. https://doi.org/10.1111/j.14698986.2008.00673.x.

Vaschillo EG, Vaschillo B, Buckman JF et al (2012) Measurement of vascular tone and stroke volume baroreflex gain. Psychophysiology 49(2). John Wiley \& Sons, Ltd (10.1111): 193-197. https://doi.org/ 10.1111/j.1469-8986.2011.01305.x

Verdejo-Garcia A, Clark L, Dunn BD (2012) The role of interoception in addiction: a critical review. Neurosci Biobehav Rev 36(8):18571869. https://doi.org/10.1016/j.neubiorev.2012.05.007

Watson P, Pearson D, Le Pelley ME (2020) Reduced attentional capture by reward following an acute dose of alcohol. Psychopharmacology. Springer: 1-15. https://doi.org/10.1007/s00213-020-05641-6

Weinstein A and Cox WM (2006) Cognitive processing of drug-related stimuli: the role of memory and attention. Journal of psychopharmacology (Oxford, England) 20(6). SAGE Publications LtdLondon, Thousand Oaks,CA and New Delhi: 850-9. https://doi.org/10.1177/ 0269881106061116

Wiers R, Field M and Stacy A (2016) Passion's slave?: conscious and unconscious cognitive processes in alcohol and drug abuse. Available at: https://books.google.es/books?hl=en\&lr=\&id=OgoDQAAQBAJ\&oi=fnd\&pg=PA311\&ots=aMDXp106Np\&sig= zw46MxeKFXxUaQWmu_e9KwG4hak (accessed 7 July 2019).

Wiers RW and Stacy AW (2006) Implicit cognition and addiction. Current Directions in Psychological Science 15(6). SAGE 
PublicationsSage CA: Los Angeles, CA: 292-296. https://oi.org/ 10.1111/j.1467-8721.2006.00455.x.

Wiers RW, Stacy AW, Ames SL, et al. (2002) Implicit and explicit alcohol-related cognitions. Alcohol Clin Exp Res 26(1). Blackwell Publishing Ltd: 129-137. https://doi.org/10.1111/j.1530-0277. 2002.tb02441.x.

Wiers RW, van Gaal S and Le Pelley ME (2021) Akrasia and addiction. Neurophilosophy and psychological mechanisms. In: Harbecke J and Herrmann-Pillath C (eds) Social Neuroeconomics. 1st ed. London: Routledge.

Publisher's note Springer Nature remains neutral with regard to jurisdictional claims in published maps and institutional affiliations. 